\title{
IDENTIFICATION OF THEILERIA SPECIES IN SHEEP AND GOATS BY THE POLYMERASE CHAIN REACTION (PCR)
}

\author{
Radwan, I.G.H. and El Kelesh, E.A. \\ Animal Health Research Institute, Dokki, Giza
}

\begin{abstract}
This study was carried out to determine the rate of Theileria infection in sheep and goats and identify the species by polymerase chain reaction (PCR). The prevalence of Theileria infection in sheep and goats was $33.75 \%$ and $28 \%$ respectively, by microscopical examination of blood smears from 80 sheep and 50 goats in Egypt. Five positive samples for Theileria infection were subjected to PCR using Theileria species specific primer. In addition, 5 negative samples for Theileria infection and apparently healthy were subjected to the same examination. Seven out of 10 blood samples (5 sheep and 2 goats) were PCR positive using Theileria species specific primer at $1098 \mathrm{bp}$. Three of those were PCR positive using T. ovis specific primer at $520 \mathrm{bp} . T$. lestoquardi was detected in 3 samples by PCR at $230 \mathrm{bp}$. Two apparently healthy animals were positive for Theileria species PCR but negative for both T. lestoquardi and T. ovis. This indicated that, other nonpathogenic Theileria species may be present in Egypt. Only one sheep sample was showed mixed infection of both T. ovis and T. lestoquardi.
\end{abstract}

\section{INTRODUCTION}

Ovine theileriosis is a tick-borne hemoprotozoan disease in sheep and goats caused by Theileria lestoquardi, T. ovis, T. separata and China Theileria species (Schnittger, 2000). Theileria lestoquardi, and China Theileria species are highly pathogenic species that cause high rate of 
morbidity and mortality while, $T$. ovis and $T$. separata were benign species which are stated to cause temporary clinical symptoms. High losses due to malignant theileriosis were recorded in indigenous sheep and goats in Iraq and Sudan with mortality rates up to $100 \%$ in case of outbreaks can occur (Friedhoff, 1997).

The classical identification of Theileria was based on morphology, host specificity, transmission mode, the vector and epidemiological data. However, different species had proved to share similar morphology under light microscopy, complete life cycle data were not available and it was difficult to obtain pure species for study (Chae et al., 1999). Diagnosis of Theileria infection was based on the light microscopy detection of parasite in thin blood smears and the presence of schizonts in stained lymph node biopsy smears (Uilenberg, 1981). However, micr-oscopic examination of Theileria from carrier animals was not always easy because low numbers of parasites led to a high false negative diagnosis, As well, diagnosis using serological tests, also had some disadvantages. The antibodies often could not be detected in long-term carriers despite the presence of piroplasms, and cross-reactivity with antibodies against other species of piroplasms was also recorded (Leemans et al. 1997). Recently, polymerase chain reaction (PCR) had allowed the development of sensitive and specific diagnostic assays for the detection of Theileria in carrier animals at low parasitaemia (Almeria rt al. 2001).

In Egypt, severe symptoms of Theileria infection as rise in temperature, enlargement of lymph nodes and abortion were observed in sheep (Harfoush, 1998-Hala and El-Kelesh 2006). Also, the clinical symptoms of theileriosis were observed in goats as rise in temperature, anorexia and corneal opacity ending by complete blindness(Arafa and Radwan 2007). 
Malignant ovine theileriosis caused by T. lestoquardi was reported in neighbours of Egypt like Sudan and Saudi Arabia. The mortality rate of ovine theileriosis reached $80 \%$ in Sudan (Salih et al. 2003) While, the morbidity were $20.46 \%$ in sheep and $7.57 \%$ in goats (El-Metenawy, 1999).

Such high rates may be an indication of the existence of $T$ lestoquardi in Egypt, and it could be the main cause of such severe symptoms observed in sheep and goats, not caused by T. ovis as it had been initially thought. Therefore, the determination of Theileria species by molecular biology has become necessary to facilitate the diagnosis, treatment and control of the disease.

The aim of the present study was to identify the Theileria species of symptomatic and asymptomatic sheep and goats in Egypt by using polymerase chain reaction (PCR) in comparison to microscopic examination of thin blood smears.

\section{MATERIALS AND METHODS}

\section{Collection of samples:}

A total of 80 sheep and 50 goats in four different provinces in Egypt were examined for theileriosis by clinical symptoms. The four provinces were: El-fayoum, Giza, Cairo and El-Gharbia. Blood samples from all sheep and goats were collected separately from each animal on anticoagulant (EDTA).

\section{Microscopic examination:}

Thin blood smears were prepared immediately after drawing the blood samples. Blood smears were fixed with methanol and stained with Giemsa. The stained blood smears were examined for Theileria piroplasms and schizonts. 


\section{Samples for PCR:}

After microscopical examination, 5 positive samples (3 from sheep and 2 from goats) for Theileria infection were subjected to PCR using Theileria species specific primer. Three of them (2sheep and one goat) showed symptoms of Theileria infection. In addition, 5 negative samples for Theileria infection and apparently healthy were subjected to the same examination. Positive samples were subjected for PCR using T. ovis specific primer and T. lestoquardi specific primer. DNA positive control for Theileria species was obtained from sheep with clinical symptoms of theileriosis, diagnosed at laboratory of parasitology department, Animal Health Research Institute, Dokki, Giza.

\section{DNA extraction:}

DNA was isolated from $200 \mathrm{ul}$ of blood. After lysis red blood cells, samples were incubated with proteinase $\mathrm{K}(200 \mathrm{ug} / \mathrm{ml})$ at $56^{\circ} \mathrm{C}$ for 1 hour. Samples were then extracted with phenol-chloroform-isoamyl alcohol (25:24:1) followed by a chloroform-isoamyl alcohol (24:1). DNA was precipitated with $100 \%$ ethanol, washed once with $70 \%$ ethanol and re-suspended in $50 \mathrm{ul}$ of Tris-EDTA before being used as template for PCR.

\section{Polymerase chain reaction (PCR) amplification:}

Three sets of primers were used and mentioned in table (1). PCR reactions were performed as described by d'Oliveira et al. (1995) with some modification for Theileria species; Altay et al. (2005) for T. ovis and Spitalska et al. (2004) for T. lestoquardi.. The reaction containing 50 $\mathrm{mM} \mathrm{kcl,} \mathrm{10m} \mathrm{M} \mathrm{tris-Hcl} \mathrm{(pH} \mathrm{8.3),} 4$ mM Mgcl2, 0.1\% Triton x100, 200 $\mathrm{uM}$ of the deoxynucleotide triphosphate (dNTPs) mix (Gibco BRL, USA), 2 units of Tag polymerase enzyme (Promega Company, Madison, 
USA), 80 Pmole of each primer and $5 \mathrm{ul}$ of processed blood samples. The reactions were performed in an automatic DNA thermal cycler (PerkinElmer, California) with a three steps cycling program as follows: 35 cycles, each cycle consisted of a denaturing step at $95^{\circ} \mathrm{C}$ for $1 \mathrm{~min}$, annealing step at $52^{\circ} \mathrm{C}$ for $1 \mathrm{~min}$ and extension at $72^{\circ} \mathrm{C}$ for $1 \mathrm{~min}$. The amplified fragments were visualized by electrophoresis in a $1.2 \%$ agarose gel containing ethidium bromide $(0.5 \mathrm{ug} / \mathrm{ml})$ run at $90 \mathrm{~V}$ for $1 \mathrm{~h}$ with 25 ul of PCR reaction loaded. A $\varphi$ X 174 RF DNA/Hae III marker (Gibco BRL, Gaithersburg, USA) was used as a molecular weight standard.

Table (1): Description of primers of Theileria sp., T.ovis and T.lestoquardi used in the study.

\begin{tabular}{||c||c||c||c||}
\hline \hline Parasite & Primers & Sequence (5_-3_) & Product size (bp) \\
\hline \hline \begin{tabular}{c|c} 
Set $(\mathrm{A})$ \\
Theileria sp. a
\end{tabular} & 989 & AGTTTCTGACCTATCAG & 1098 \\
\hline Set (B) & TSst170F & TTGCCTTAAACTTCCTTG & \\
Theileria ovis b & TSst670R & TCCGGACACCTTCGGGT & 520 \\
\hline Set (c) & F2 & GACACAGGGAGGTAGTGACAAG & 230 \\
Ttheileria & R2 & CTAAAGAATTTCACCTTTCTGACA & \\
lestoquardi c & & & \\
\hline
\end{tabular}

a As described by d'Oliveira et al. (1995)

b As described by Altay et al. (2005)

c As described by Spitalska et al. (2004)

\section{RESULTS}

\section{Microscopical results:}

Microscopical examination of blood smears showed that, overall, the prevalence of Theileria infection in sheep and goats was recorded as $33.75 \%$ and $28 \%$ respectively. Piroplasms and schizonts (macro and micro) stages were detected in both sheep and goats (Figs. 1 and 2 a \& 
b). Table (2) showed that, Theileria infection was more detected in sheep and goats in El-Fayoum Governorate, followed by El- Gharbia Governorate in case of sheep while, the second province in case of goats was Giza. Only 12 sheep and 8 goats of these 27 and 14 microscopically positive showed symptoms respectively, such as fever, enlargement of lymph nodes and corneal opacity.

Table (2): Prevalence of Theileria infection in sheep and goats by microscopical examination.

\begin{tabular}{|c|c|c|c|c|c|c|c|c|}
\hline \multirow{2}{*}{ provinces } & \multicolumn{2}{|c|}{$\begin{array}{c}\text { Numbers of examined } \\
\text { animals }\end{array}$} & \multicolumn{2}{|c|}{ Sheep } & \multicolumn{2}{|c|}{$\begin{array}{c}\text { Numbers of examined } \\
\text { animals }\end{array}$} & \multicolumn{2}{|c|}{ goats } \\
\hline & symptomatic & $\begin{array}{l}\text { Asympto- } \\
\text { matic }\end{array}$ & $\begin{array}{l}\text { Positive } \\
\text { with ME }\end{array}$ & $\%$ & symptomatic & asymptomatic & $\begin{array}{l}\text { Positive } \\
\text { with ME }\end{array}$ & $\%$ \\
\hline El-fayoum & 7 & 10 & 9 & $52 \%$ & 1 & 7 & 3 & $37.5 \%$ \\
\hline Giza & 0 & 25 & 4 & $16 \%$ & 7 & 23 & 9 & $30 \%$ \\
\hline Cairo & 0 & 20 & 5 & $25 \%$ & 0 & 0 & 0 & 0 \\
\hline El-Garbia & 5 & 13 & 9 & 9 & 0 & 12 & 2 & 10 \\
\hline total & 12 & 68 & 27 & $33.5 \%$ & 8 & 42 & 14 & $28 \%$ \\
\hline
\end{tabular}

ME: microscopic examination

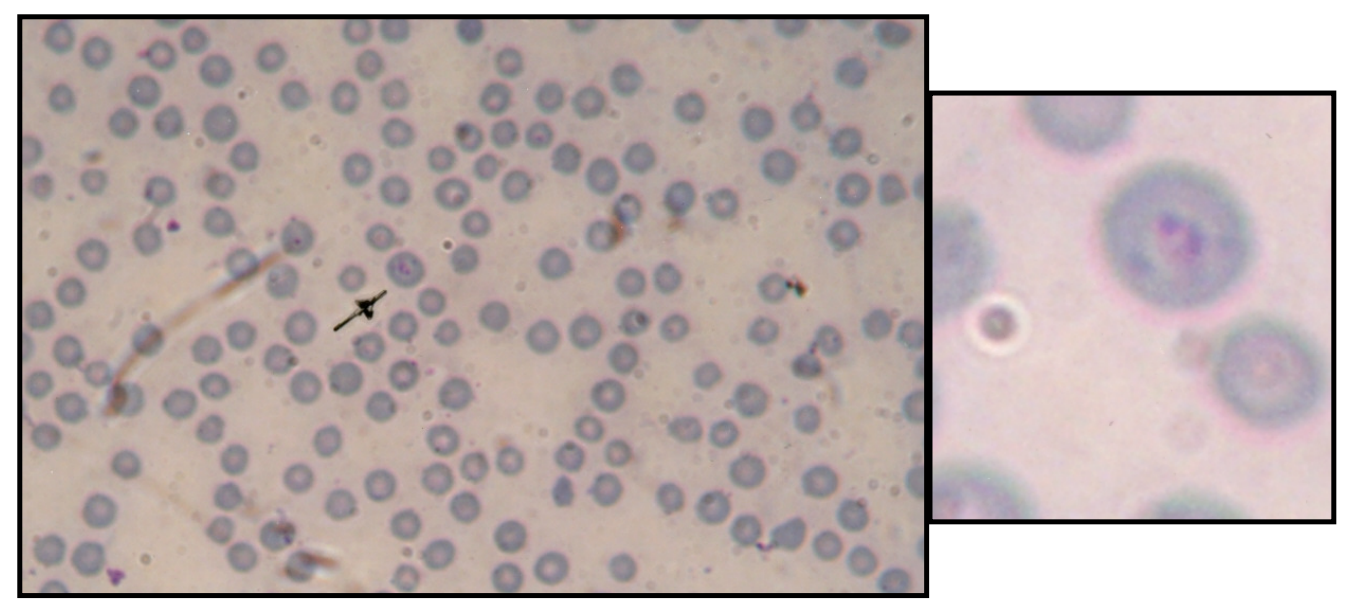

Fig. (1): Erythrocytic stage of Theileria in sheep and goats. 


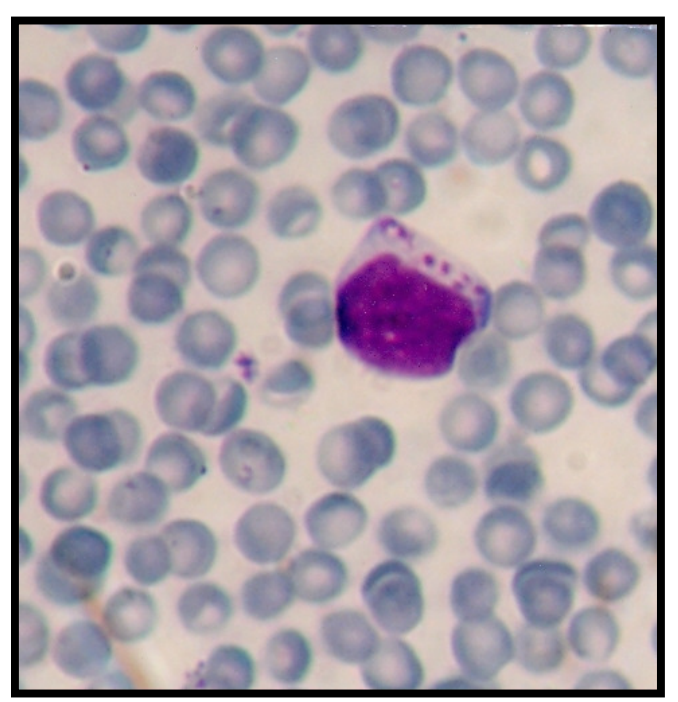

(A)

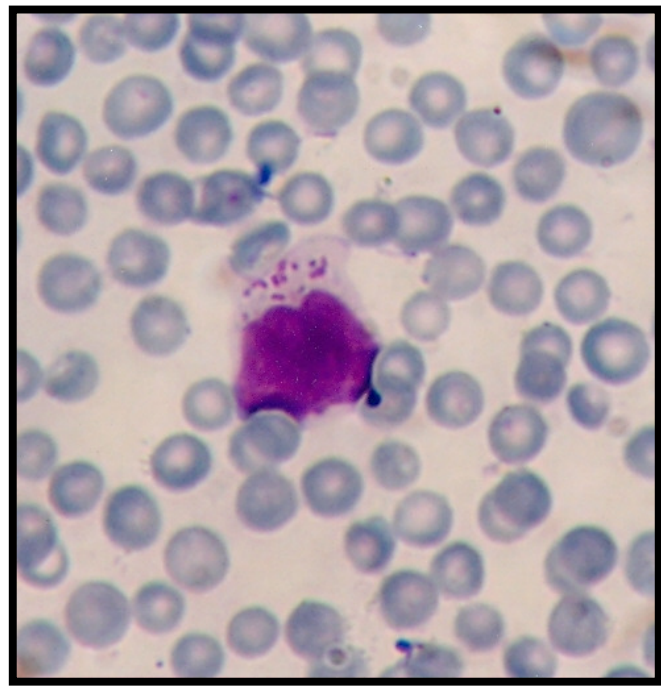

(B)

Fig. (2): Schiont stage in lymphocytes of sheep and goats:

a- Macroschizont

b- Microschizont

\section{PCR results:}

Table (3) showed that, all samples proved to be positive by thin blood smears examination were also positive by PCR after using Theileria species specific primer. Whereas, no piroplasms and schizont stages were seen in 2 samples by microscopical examinations were proved to be positive by PCR. Seven out of 10 blood samples (5 sheep and 2 goats) were PCR positive using Theileria species specific primer at 1098 bp (Fig.3). Three of those (apparently healthy) were PCR positive using $T$. ovis specific primer at $520 \mathrm{bp}$ (Fig. 4). T. lestoquardi was detected in 3 animals showed clinical symptoms of theileriosis (2 sheep and 1 goat) by PCR at 230 bp (fig. 5). Only one sheep sample showed mixed infection of both $T$. ovis and T. lestoquardi. 
Radwan, I.G.H. \& El Kelesh, E.A.

Table (3): The results of PCR in comparison with microscopical examination.

\begin{tabular}{|c|c|c|c|c|c|c|}
\hline \multirow{2}{*}{$\begin{array}{l}\text { No. of } \\
\text { animal }\end{array}$} & \multirow{2}{*}{ Animals } & \multirow{2}{*}{$\begin{array}{c}\text { Clinical } \\
\text { symptoms }\end{array}$} & \multirow{2}{*}{ ME } & \multicolumn{3}{|c|}{ PCR } \\
\hline & & & & $\begin{array}{c}\text { Theileria } \\
\text { species }\end{array}$ & T. ovis & T.lestoquardi \\
\hline 1 & Sheep & + & + & + & - & + \\
\hline 2 & Sheep & + & + & + & + & + \\
\hline 3 & Sheep & - & + & + & + & - \\
\hline 4 & Goat & + & + & + & - & + \\
\hline 5 & Goat & - & + & + & + & - \\
\hline 6 & Sheep & - & - & + & - & - \\
\hline 7 & Sheep & - & - & + & - & - \\
\hline 8 & Sheep & - & - & - & - & - \\
\hline 9 & Goat & - & - & - & - & - \\
\hline 10 & Goat & - & - & - & - & - \\
\hline Total & & 3 & 5 & 7 & 3 & 3 \\
\hline
\end{tabular}

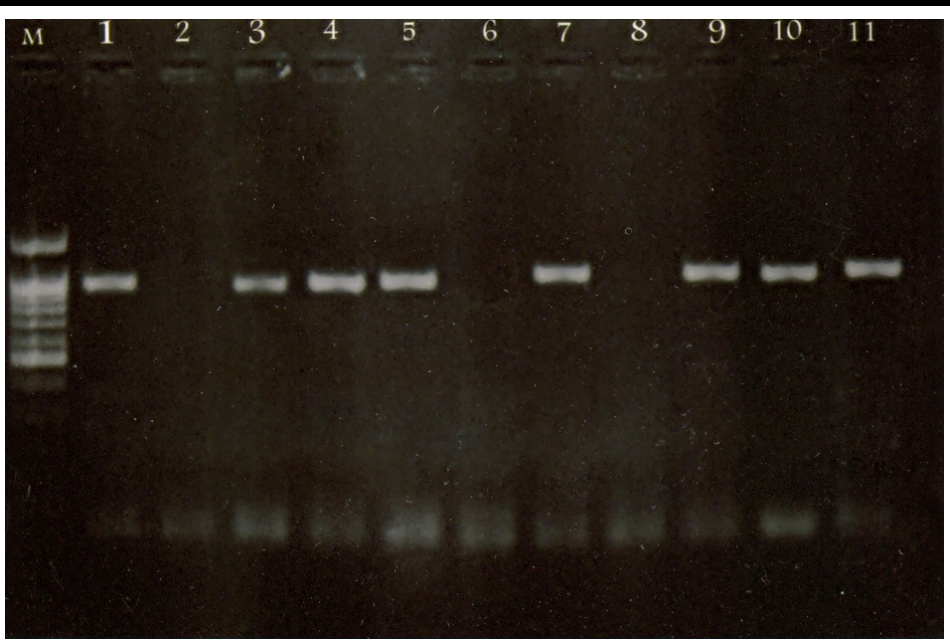

Fig. (3): Agarose-gel electrophoresis of amplification products obtained from genomic DNA of Theileria species of sheep and goats (1098bp PCR product) by using primer set $\mathrm{A}$.

Lanes: M: molecular weight standards (100bp), Lane 1: positive control;

Lane 2-11: Theileria species isolated from sheep and goats. 


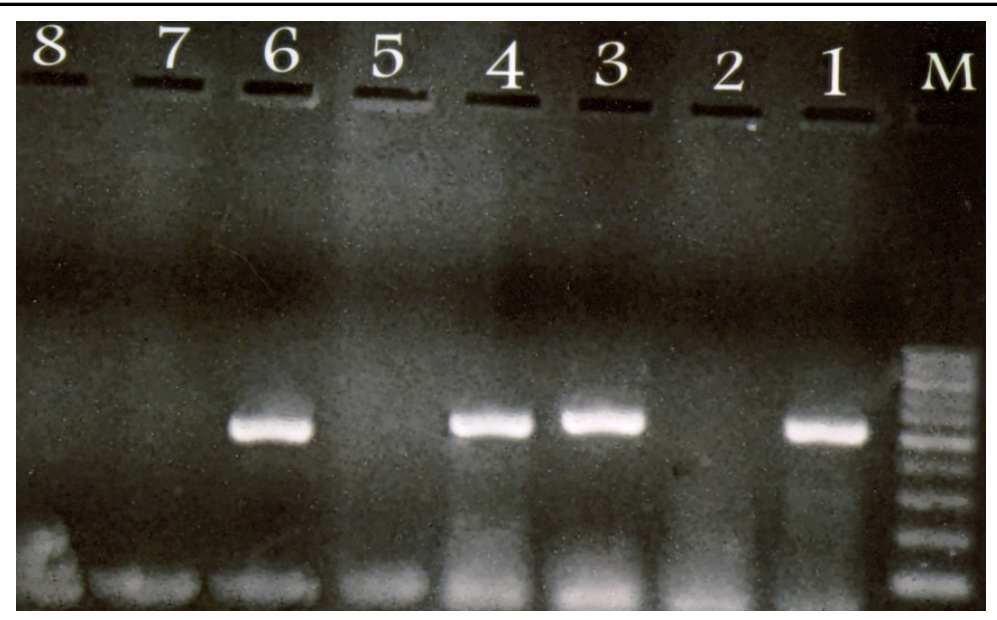

Fig. (4): Agarose-gel electrophoresis of amplification products obtained from genomic DNA of Theileria species of sheep and goats using T. ovis specific primer (520bp PCR product). Lanes: M: molecular weight standards (100bp), Lane 1: positive control; Lane 2-7: Theileria species isolated from sheep and goats.

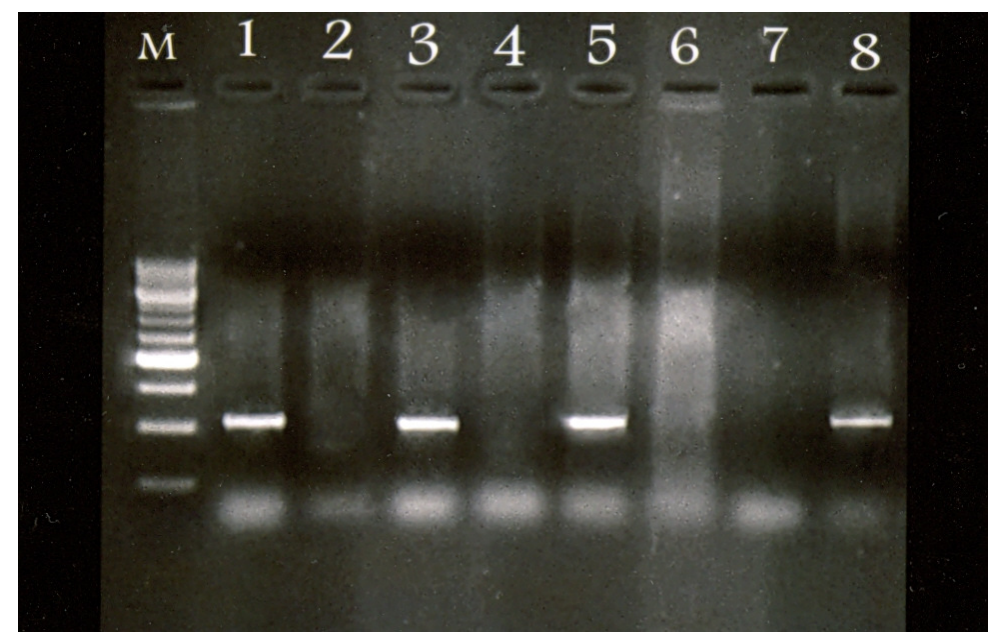

Fig. (5): Agarose-gel electrophoresis of amplification products obtained from genomic DNA of Theileria species of sheep and goats using $T$. lestoquardi specific primer (520bp PCR product). Lanes: M: molecular weight standards (100bp), Lane 1: positive control; Lane 2-7: Theileria species isolated from sheep and goats. 


\section{DISCUSSION}

Theileriosis is an important parasitic disease of sheep and goats in Egypt which causes great economic losses of animals and their products. In the present study, the prevalence of Theileria infection in sheep and goats was detected at rate of $33.75 \%$ in sheep and $28 \%$ in goats by microscopic examination of blood smears. These results indicated a relatively high rate of Theileria infection in the 4 provinces especially El-Fayoum Governorate. Therefore, strict control of ticks, life-long treatment and development of vaccine for theileriosis become necessary. These percentages are approximately near to those recorded in Egypt by Harfoush,(1998) 58.2\% in sheep, Hala and El-Kelesh (2006) $87.5 \%$ and $18.75 \%$ in sheep on farms in El-Gharbia and slaughter-houses respectively. Arafaa and Radwan (2007) recorded a percentage rate of $86.1 \%$ in goats showing clinical symptoms of theileriosis and $43.1 \%$ of apparently healthy in Giza Governorate. They stated that, Theileria species found in Egypt might be $T$. lestoquardi or/and T. ovis. Several Theileria species can cause theileriosis in sheep and goats. Of these; T. lestoquardi which is considered to be the most highly pathogenic; the other species of Theileria such as T.ovis were less pathogenic and had lower economic importance than T. lestoquardi (Uilenberg, 1981). It is difficult to differentiate these two species on the basis of morphology of piroplasm and schizont stages especially in mixed infection. Also, Theileria species of sheep and goats can be detected routinely by conventional microscopic examination, but it lacked sensitivity and inconsistency of results, especially in asymptomatic or carrier animals since the parasitemia level was very low (El-Zeedy et al.(1998). 
In the present study, PCR was used for discrimination T. lestoquardi from T. ovis and other Theileria species of sheep and goats. Seven out of 10 sheep and goats were positive for Theileria species specific primer (set A) at 1098 bp. These results are supported by d'Oliveira et al (1995) who used Theileria species specific primer (989 and 990), deduced from small subunit (SSUrRNA) gene for amplification of the expected 1098 bp DNA fragment from all Theileria species samples examined.

Because Theileria species primer (set A) could not differentiate between $T$. lestoquardi and T. ovis, the usage of specific primers for $T$. ovis (set B) and T. lestoquardi (set C) were necessary. Three blood samples of those proved to be positive with primer set A gave the expected 520 bp amplified PCR product with primer set B. This result indicated that primer set B was specific for $T$. ovis. This finding was in agreement with Altay et al. (2005) who used T. ovis specific primer in the amplification of expected $520 \mathrm{bp}$ for diagnosing T. ovis in sheep.

T. lestoquardi was detected in three animals ( 2 sheep and one goat) out of those proved to be positive with primer set (A) using primer set (C) at $230 \mathrm{bp}$. This result proved the presence of the pathogenic Theleria species (T. lestoquardi) in Egypt (El-Fayoum, El-Gharbia and Giza). This result was confirmed by that result obtained by Spitalska et al (2004). Thus, 2 animals without exhibiting any clinical symptoms were positive for Theileria species PCR but negative for both T.lestoquardi and T.ovis. This indicated that, other non-pathogenic Theileria species may be present in Egypt. 
The results obtained from this study indicated that, the highest sensitivity of PCR-based techniques compared to blood smear examinations in the diagnosis of theileriosis in sheep and goats as shown by the fact that, two blood samples were demonstrated positive for Theileria infection by PCR amplification while, were negative result by microscopic examinations. This result was explained by Altay et al.(2005) who found that sensitivity of PCR for T. ovis was equal to a parasitemia at the level of $10^{-5}$ and was over 100 times greater than can be attained by microscopic examination of Giemsa stained thin blood smears. Also, Almeria et al. (2001) confirmed the previous results, by the using of PCR, this resulted in significantly higher efficacy of detection of Theilria species in cattle compared to microscopical observation of blood smears and allowed the specific discrimination between pathogenic and non-pathogenic Theileria.species.

The non detectable PCR amplification in three blood samples came concurrently with the negative results by microscopic examination.

In conclusion, the results obtained in this study suggested that, The using of PCR Theileria specific primers was a highly sensitive diagnostic technique, for exact discrimination between pathogenic ( $T$. lestoquardi) and non-pathogenic (T. ovis) Theileria species in sheep and goats. This will be useful tools for clarifying the epidemiology of ovine theileriosis in Egypt, so help to establish adequate control programmes for tick eradication in Egypt.

\section{REFERENCES}

- Altay, K., Dumanli, N., Holman, P.J.and Aktas, M. (2005): Detection of Theileria ovis infected sheep by nested PCR. Vet Parasitol 127:99104. 
- Almeria, S., Castellà, J., Ferrer, D., Ortuo, A., Estrada-Pea,A. and Gutiérrez,J.F.(2001): Bovine piroplasms in Minorca(Balearic Islands, Spain): a comparison of PCR-based and light microscopy detection. Vet. Parasitol. 99, 249-259.

- Arafaa, M.M. and Radwan, I.G.H. (2007): Parasitological, haematological and biochemical studies on naturally infected goat with Theileria species in Egypt. Vet. Med. J. 55 (4): 1087-1099.

- Chae, J.S., Allsopp, B.A., Waghela, S.D., Park, J.H., Kakuda, T., Sugimoto, C., Allsopp, M.T., Wagner, G.G.and Holman, P.J. (1999): A study of the systematics of Theileria spp based upon small-subunit ribosomal RNA gene sequences. Parasitol Res. 85:877-883.

- d'Oliveira, C., Van der Weide, M., Habela, M.A., Jacquiet, P. and Jongejan, F.(1995): Detection of Theileria annulata in blood samples of carrier cattle by PCR. J. Clin Microbiol 33:2665-2669.

- El-Metenawy, M. (1999): Blood Parasites of sheep and goats at AIQassim Region, Saudi Arabia. J. Protozoo!. Kes.. 9. 71 - 75

- El-Zeedy,S.A., Farah, A.W. and Abbas, A.M. (1998): Detection of Theileria annulata carrier cattle in Egypt by the polymerase chain reaction. Assiut Vet. Med. J. 38 (76) 251-263.

- Friedhoff, K.T.(1997): Tick-borne diseases of sheep and goats caused by Babesia, Theileria or Anaplasma spp. Parasitologia 39:99-109

- Hala,H. W. and El-Kelesh, E.A (2006): Haematological and Biochemical Changes in blood of Sheep suffering from Theileria infection. Egypt.J. Animal and Poultry Management, 1 (1): 201-217. 
- Harfoush, M.A. (1998): Studies on some parasitic diseases in sheep. Ph.D. thesis Tanta University, faculty of Veterinary Medicine, Animal diseases department.

- Leemans, I., Hooshmand-Rad, P. and Uggla, A. (1997): The indirect fluorescent antibody test based on schizont antigen for study of the sheep parasite Theileria lestoquardi. Vet Parasitol 69:9-18.

- Salih, D.A., ElHussein, A.M., Hayat, M. and Taha, K.M. (2003): Survey of Theileria lestoquardi antibodies among Sudanese sheep. Vet Parasitol. 27;111(4):361-7.

- Schnittger, L., Yin, H., Jianxun, L., Lugwing, W., Shayan, P., Rahbari, S., Voss-Holtmann, A. and Ahmed, J.S. (2000): Ribosomal small-subunit RNA gene-sequence analysis of Theileria lestoquardi and a Theileria species highly pathogenic for small ruminants in China. Parasitol Res 86:352-358

- Spitalska,E., Torina,a., Cannella, V., Caracappa, S. and Olivier, A.E. (2004): Discrimination between Theileria lestoquardi and Theileria annulata in their vectors and hosts by RFLP based on the 18SrRNA gene. Parasitol. Res. 10 1217-2.

- Uilenberg, G., (1981): Theilerial species of domestic livestock. In: Irvin A.D., Cunningham M.P., Young, A.S. (Eds.), Advances in the Control of Theileriosis. Martinus Nijhoff Publishers, The Hague, pp. 4-37. 